\title{
THE USAGE OF THE TREFFTZ METHOD TO DETERMINE THE BIOT NUMBER*
}

\author{
Artur Maciag ${ }^{1}$, Magdalena Walaszczyk ${ }^{2}$ \\ ${ }^{I}$ Faculty of Management and Computer Modelling, Kielce University of Technology \\ Kielce, Poland \\ ${ }^{2}$ Faculty of Mechatronics and Machine Design, Kielce University of Technology \\ Kielce, Poland \\ maciag@tu.kielce.pl,mwalaszczyk@tu.kielce.pl
}

Received: 7 November 2017; Accepted: 18 December 2017

\begin{abstract}
The paper presents a method for determining the Biot number and the heat transfer coefficient based on the Trefftz functions. Firstly, the temperature distribution in the entire domain is calculated and then used for obtaining the heat transfer coefficient. The usefulness of the method is shown in the examples. The data for the examples are calculated by means of a known exact solution or they are given as measurements. The sensitivity of the presented method is checked. Test examples are used to check the method. Next, the heat transfer coefficient is determined for the real data for a rocket engine.
\end{abstract}

MSC 2010: $35 \mathrm{~K} 05,65 \mathrm{M} 32$

Keywords: Biot number, Trefftz functions, data disturbance, inverse problem

\section{Introduction}

The heat transfer coefficient plays a significant role in many engineering problems. It also enables us to construct algorithms for optimizing production processes. A tool that supports the optimization procedures is a numerical simulation of the heat transfer process. The simulation is possible with knowledge of the boundary conditions of heat exchange and especially knowledge of the heat transfer coefficient. Determination of this coefficient is difficult due to the necessity of placing sensors on the surface of the machine, which can interfere the heat transfer. Sometimes installing a sensor on the surface is impossible.

Determination of the heat transfer coefficient belongs to the class of inverse problems. These problems can be solved by means of the Trefftz method. The fundamentals of the Trefftz method were presented by Erich Trefftz in 1926 [1].

\footnotetext{
* This topic was presented at the conference Numerical Heat Transfer in Warsaw, 27-30 September 2015.
} 
In general it is addressed to solving linear differential equations. However, the method can be also applied to solve nonlinear direct and inverse problems. At first, the non-stationary problems were reduced to stationary ones by the discretization of the time. In 1956, Rosenbloom and Widder considered the time as a continuous variable [2]. This approach was then applied for solving various partial differential equations [3]. This method was also used to determine the temperature field and heat transfer coefficient in the contact between heating foil and fluid in flow boiling in a vertical minichannel [4]. The paper [5] presents the determination of the Biot number and the heat transfer coefficient using the Laplace's transform techniques, on the basis of experimental data. The same experimental data were used by Bartz [6] and Mehta [7,8] to determine the heat transfer coefficient. In this paper, the Trefftz functions have been used in order to identity the coefficient. Results have been based on the experimental data presented in [7] and compared with results obtained in the papers [5-8] where the same input data were taken into consideration.

\section{Application of the Trefftz method}

In heat conduction problems, the temperature field is determined by the differential equation of heat conduction and initial and boundary conditions. The dependence of the parameters describing the boundary conditions of time allows us to determine the temperature distribution inside the domain by solving the heat conduction equation. This type of problem is called a direct problem. In many cases, the boundary conditions such as heat flux density, the temperature of the surface, the heat transfer coefficient or the ambient temperature are not known. Instead of that, we can measure the temperature of selected points within the body and, on that basis, determine the time dependent temperature field and coefficient. Such problems are called inverse problems, and these problems are usually ill-posed. Therefore, we should check how the data disturbances affect on the obtained results.

The Trefftz method is addressed to solving linear differential equation:

$$
L u(\boldsymbol{x}, t)=f(\boldsymbol{x}, t) \quad \text { for }(\boldsymbol{x}, t) \in \Omega \times\langle 0, \infty),
$$

where $L$ is a linear partial differential operator and $\Omega$ is a bounded subset of $R^{n}$. The equation should be completed by appropriate initial and boundary conditions:

$$
\begin{gathered}
I u(\boldsymbol{x}, 0)=h(\boldsymbol{x}) \quad \text { for } \boldsymbol{x} \in \Omega, \\
B u(\boldsymbol{x}, t)=g(\boldsymbol{x}, t) \quad \text { for }(\boldsymbol{x}, t) \in \partial \Omega \times\langle 0, \infty),
\end{gathered}
$$

where $\partial \Omega$ is a boundary of $\Omega$. 
If the Trefftz functions for operator $L$ are known, the solution can be approximated by:

$$
u(\boldsymbol{x}, t) \approx w(\boldsymbol{x}, t)=\sum_{n=1}^{N} c_{n} V_{n}(\boldsymbol{x}, t)
$$

where $c_{n}$ are coefficients of the linear combination and $V_{n}$ are Trefftz functions for linear operator $L$. The coefficients of the linear combination are calculated based on known initial and boundary conditions. For this purpose a suitable functional, which describes the error of fulfilling the initial and boundary conditions by approximate solution in least square sense, has to be minimized. After obtaining the temperature distribution in the entire domain, the heat transfer coefficient can be calculated using the Robin boundary condition.

\section{Example of the problem with the data disturbance}

The method presented in this article is illustrated by a suitable example. To check the quality of the approximation, test problems were chosen for which the exact solutions are known.

Let us consider the one dimensional heat conduction equation:

$$
\left(\frac{\partial^{2}}{\partial x^{2}}-\frac{\partial}{\partial t}\right) T(x, t)=0 \text { for } x \in(0,1), t>0,
$$

with initial and boundary conditions:

$$
\begin{gathered}
T(x, 0)=e^{-x}, \\
T(0, t)=e^{t} .
\end{gathered}
$$

Moreover, the values of the temperature in 11 instants on the border $x=0$ for the time between 6 and 16 seconds are known. They are called the internal responses. In this example, the values of the internal responses were calculated by means of an exact solution. In practice, they are given as measurements.

The exact solution of a given problem is known and has a form:

$$
T(x, t)=e^{t} e^{-x} .
$$

In this case, the inverse problem is considered (two conditions for $x=0$ and lack of condition for $x=1$ ). For numerical simulation, the exact solution has been taken into account.

The presented problem was solved by the Trefftz method. There are several methods of determining the Trefftz function. In this case, the Trefftz functions are heat polynomials. They were introduced in $[2,3]$. The first 8 Trefftz functions are as follows: 


$$
\begin{gathered}
V_{1}=1, \\
V_{2}=x, \\
V_{3}=t+\frac{1}{2} x^{2}, \\
V_{4}=x t+\frac{1}{6} x^{3} \\
V_{5}=\frac{1}{2} t^{2}+\frac{1}{2} x^{2} t+\frac{1}{24} x^{4}, \\
V_{5}=\frac{1}{2} t^{2}+\frac{1}{2} x^{2} t+\frac{1}{24} x^{4}, \\
V_{6}=\frac{1}{2} x t^{2}+\frac{1}{6} x^{3} t+\frac{1}{120} x^{5}, \\
V_{7}=\frac{1}{6} t^{3}+\frac{1}{4} x^{2} t^{2}+\frac{1}{720} x^{6}+\frac{1}{24} x^{4} t, \\
V_{8}=\frac{1}{120} x^{5} t+\frac{1}{6} x t^{3}+\frac{1}{5040} x^{7}+\frac{1}{12} x^{3} t^{2} .
\end{gathered}
$$

To determine the coefficients of the linear combination $c_{n}$, the following functional should be minimized:

$$
\begin{aligned}
\int_{0}^{1}\left(T_{a}(x, 0)-\right. & T(x, 0))^{2} d x+\int_{0}^{16}\left(T_{a}(0, t)-T(0, t)\right)^{2} d t+ \\
& +\sum_{i=6}^{16}\left(T_{a}\left(0, t_{i}\right)-T\left(0, t_{i}\right)\right)^{2} .
\end{aligned}
$$

After obtaining the temperature distribution in the entire domain, we can calculate the Biot number using the Robin condition in the form:

$$
\left(\frac{\partial T}{\partial x}(1, t)\right)=-B i\left(T(1, t)-\frac{1}{3}\right)
$$

We can also obtain the value of the heat transfer coefficient using its relationship with the Biot number:

$$
\alpha=\frac{B i \lambda}{h}
$$

where $\alpha$ is the heat transfer coefficient, $B i$ is the Biot number, $\lambda$ is thermal conductivity and $h$ is characteristic length.

Generally, problems based on measurement data can be very sensitive to the disturbance of the input data. The measurement errors are usually the result of interpolation of measuring instruments, the uncertainty associated with the calibration and fluctuations in the sensor reading during the measurement. Therefore, 
the data disturbance sensitivity of the presented method has to be checked. Let us assume that the internal responses $u\left(0, t_{i}\right)$ are disturbed by a random error $\delta$, which has a normal distribution with a mean equal to 0 and a standard deviation of $0.01,0.02,0.05,0.07$ and 0.09 :

$$
u\left(0, t_{i}\right)=(1+\delta) \cdot u\left(0, t_{i}\right) \text { for } i \in\{6,7, \ldots, 16\} .
$$

This means that the average disturbance of the data is at the level of 1,2,5,7 and $9 \%$ respectively.

Because the exact solution is known, the quality of the approximation of the boundary condition $x=1$ can be checked by calculating a mean relative error:

$$
E=\sqrt{\frac{\int_{0}^{16}\left[T_{a}(1, t)-T(1, t)\right]^{2} d t}{\int_{0}^{16}[T(1, t)]^{2} d t}} \cdot 100 \%
$$

where $T_{a}$ is the approximate solution and $T$ is the exact solution. The values of the errors with and without the disturbance data depending on the number of heat polynomials used in approximation are presented in Table 1.

Table 1

A mean relative error of the boundary temperature approximation [\%] depending on the number of the Trefftz functions and standard deviation

\begin{tabular}{|c|c|c|c|c|c|c|}
\hline \multirow{2}{*}{$\begin{array}{c}\text { Number of } \\
\text { functions }\end{array}$} & \multirow{5}{*}{ Mean relative error [\%] } \\
\cline { 3 - 7 } & Exact data & \multicolumn{5}{|c|}{ Disturbed data (standard deviation) } \\
\cline { 3 - 7 } & & 0.01 & 0.02 & 0.05 & 0.07 & 0.09 \\
\hline 5 & $2.59 \%$ & $2.46 \%$ & $5.09 \%$ & $8.94 \%$ & $11.53 \%$ & $14.12 \%$ \\
6 & $0.84 \%$ & $1.06 \%$ & $4.21 \%$ & $11.67 \%$ & $16.65 \%$ & $21.63 \%$ \\
7 & $0.21 \%$ & $0.89 \%$ & $3.71 \%$ & $9.01 \%$ & $12.55 \%$ & $16.09 \%$ \\
8 & $0.07 \%$ & $4.309 \%$ & $4.13 \%$ & $10.95 \%$ & $15.49 \%$ & $20.05 \%$ \\
\hline
\end{tabular}

Clearly, the Trefftz method gives very good results. In general, more Trefftz functions lead to better results. In this problem, the error of approximation is very small even for a small number of functions. The further increase in the number of heat polynomials causes an accumulation of numerical errors and worse results. Generally, for disturbed data the error is bigger. However, it should be noted that the disturbances of the internal responses do not cause a considerable increase of the error. Of course, greater disturbance results in bigger errors. The values presented in Table 1 show that even for a disturbance of $2 \%$, the errors are on the level of $4 \%$. For greater disturbance, the errors do not exceed a double disturbance. In the case where there is no disturbance of the data, the best results are obtained for 8 Trefftz functions and in the case where we consider the disturbance for 5 or 7 functions. 


\section{Example of the problem with the experimental data}

The second example shows the usage of the presented method for calculating the heat transfer coefficient and the Biot number using experimental data. They concern the measurement of temperature on the outer wall of the divergent nozzle rocket engine, made during testing of the engine. The nozzle of the rocket engine is responsible for the conversion of pressure resulting in a jet propulsion engine. The nozzle is designed to increase the speed of gases produced in the combustion process and the ejection of them which generates kinetic energy to drive the rocket. In the divergent part of the nozzle, exhaust gases during the expansion are additionally accelerated to supersonic speed, which significantly increases the engine power. The wall thickness of the nozzle $h=0.0211 \mathrm{~m}$ enables us to treat it as an approximately flat slab.

Therefore, let us consider the equation:

$$
\left(\frac{\partial^{2}}{\partial x^{2}}-\frac{\partial}{\partial t}\right) T(x, t)=0 \text { for } x \in(0,1), t>0 .
$$

The equation should be completed by the initial boundary conditions:

$$
\begin{aligned}
& T(x, 0)=0, \\
& T(0, t)=0 .
\end{aligned}
$$

Additionally, we know the temperature on the outer wall of the divergent nozzle rocket engine in 11 instants for the time between 6 and 16 seconds:

$$
T\left(0, t_{i}\right)=T_{i}, i=1, \ldots 11
$$

Other necessary data are as follows: the ambient temperature of the nozzle $T_{o}=300 \mathrm{~K}$, gas temperature in the nozzle $T_{g}=2946.2 \mathrm{~K}$, density $\rho=7900 \mathrm{~kg} / \mathrm{m}^{3}$, specific heat $c_{p}=545 \mathrm{~J} / \mathrm{kgK}$, thermal conductivity $\lambda=35 \mathrm{~W} / \mathrm{mK}$.

This problem resembles the previous example but is based on the measurement data. Therefore it has been solved by means of Trefftz functions, as shown in the example above. After obtaining the temperature distribution in the entire domain, the Biot number was calculated using the formula:

$$
\left(\frac{\partial T}{\partial x}(1, t)\right)=-B i(T(1, t)-1) \text {. }
$$

Since in the first example the smallest errors were observed for 8 Trefftz functions, to solve the second problem, 8 heat polynomials have also been used.

This problem has been solved by many researchers using a variety of methods. The obtained values of the heat transfer coefficient by different methods are presented in Table 2 . 
In Table 2, the results obtained using the first five methods have been taken from literature. Methods 1 and 2 were presented by Grysa in [5]. The results of the third method are taken from Bartz article [6]. Mehta in papers [7] and [8] presented the results of methods 4 and 5 respectively. The last column shows the results obtained by the Trefftz method for 8 heat polynomials.

When comparing different methods we can see a relatively large variation of the results. This is probably related to the specifics of each method, meaning certain simplifications and calculation methods. The results obtained by the presented method seem to be correct in the physical sense. The value of the heat transfer coefficient increases with increasing temperature. There is no such relationship in the case of the methods 1, 3 and 4 . In beginning of the first method, the heat transfer coefficient increases and then starts to decrease after 12 seconds. The third method is characterized by a constant value of the coefficient which probably results from the accepted model simplifications. A decrease in the heat transfer coefficient with increasing temperature seems even more incorrect. In the second method, we observe an increase in the coefficient value and then stabilization after 12 seconds at the level of $1130 \mathrm{~W} / \mathrm{m}^{2} \mathrm{~K}$. In the fifth method, there is a similar trend as in the case of the Trefftz method, but the results obtained by the Trefftz method are about two times greater. If we take into consideration a mean of methods $1,2,3,4$ and 5 we obtain the values similar to the Trefftz method. A big advantage of the Trefftz method is the fact that the approximate solution strictly satisfies the governing equation.

Table 2

The values of the heat transfer coefficient obtained by various methods

\begin{tabular}{|c|c|c|c|c|c|c|c|c|}
\hline \multirow{3}{*}{$\begin{array}{c}\text { Time } \\
{[\mathrm{s}]}\end{array}$} & \multirow{3}{*}{$\begin{array}{c}\text { Temperature } \\
{[\mathrm{K}]}\end{array}$} & \multicolumn{7}{|c|}{ Heat transfer coefficient $\alpha$} \\
\hline & & \multicolumn{6}{|c|}{ Other methods } & \multirow[b]{2}{*}{$\begin{array}{l}\text { Trefftz } \\
\text { method }\end{array}$} \\
\hline & & 1 & 2 & 3 & 4 & 5 & $\begin{array}{c}\text { Mean of } \\
\text { methods } \\
1,2,3,4,5\end{array}$ & \\
\hline 6 & 324.69 & 738.8 & 239.5 & 2254.2 & 1821.9 & 536.6 & 1118.2 & 953.3 \\
\hline 7 & 342.02 & 1074.2 & 696.6 & 2254.2 & 1810.0 & 600.6 & 1287.12 & 993.2 \\
\hline 8 & 355.99 & 1175.7 & 884.3 & 2254.2 & 1610.3 & 592.6 & 1303.42 & 1036.4 \\
\hline 9 & 379.92 & 1241.7 & 969.5 & 2254.2 & 1690.9 & 674.2 & 1366.1 & 1083.3 \\
\hline 10 & 402.01 & 1291.6 & 1056.9 & 2254.2 & 1669.7 & 712.9 & 1397.06 & 1134.0 \\
\hline 11 & 425.01 & 1318.9 & 1111.7 & 2254.2 & 1641.9 & 737.4 & 1412.82 & 1189.0 \\
\hline 12 & 440.01 & 1300.4 & 1138.5 & 2254.2 & 1497.6 & 718.2 & 1381.78 & 1248.5 \\
\hline 13 & 459.99 & 1279.6 & 1133.6 & 2254.2 & 1443.1 & 723.6 & 1366.82 & 1313.1 \\
\hline 14 & 478.99 & 1257.6 & 1129.0 & 2254.2 & 1387.0 & 723.0 & 1350.16 & 1383.3 \\
\hline 15 & 507.01 & 1255.2 & 1127.0 & 2254.2 & 1413.0 & 753.6 & 1360.6 & 1459.5 \\
\hline 16 & 527.97 & 1248.5 & 1135.5 & 2254.2 & 1383.7 & 758.3 & 1356.04 & 1542.5 \\
\hline
\end{tabular}

If we know the value of the heat transfer coefficient in subsequent moments of time, we can use formula (11) to calculate the value of the Biot number. Figure 1 shows the relationship between the heat transfer coefficient and the Biot number. 
a)

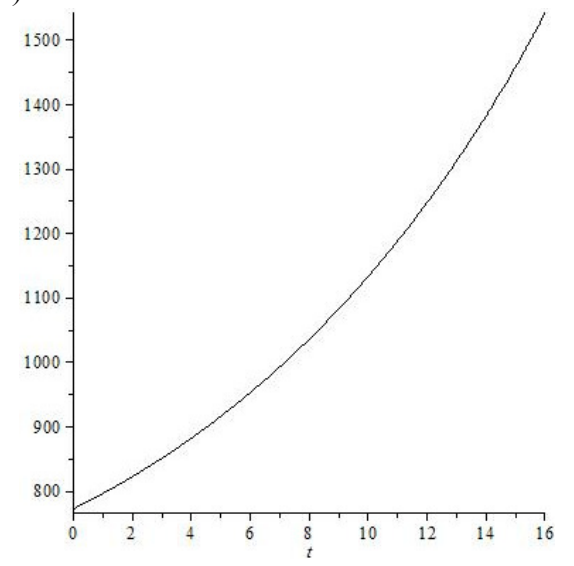

b)

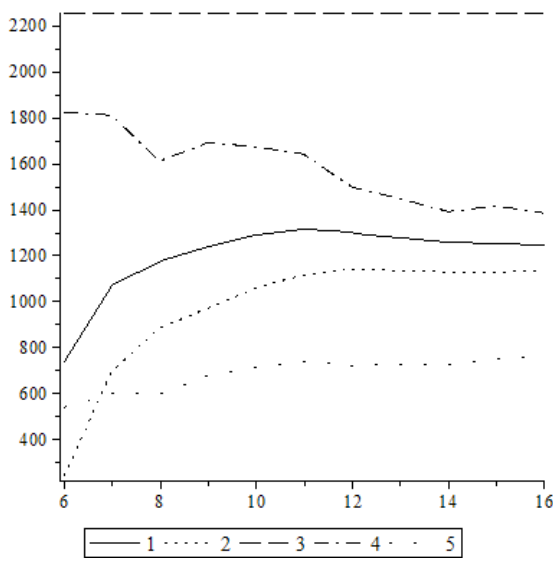

Fig. 1. The dependence of the heat transfer coefficient on time for Trefftz method (a) and for other methods (b)

In the analyzed example, the dependence of the heat transfer coefficient and the Biot number from time is characterized by the same shape. However, the values of the Biot number are about 1650 times lower than the values of the heat transfer coefficient. The Trefftz method gives a more regular graph than other methods.

\section{Conclusions}

The Trefftz method is very useful for many theoretical and practical problems of heat transfer. It gives very good results in the case of inverse problems, also concerning the identification of coefficients. It allows us to calculate the heat transfer coefficient and the Biot number when it is not possible to measure surface temperature, or when the measurement is very difficult.

A big advantage of this method is its mathematical simplicity. Moreover, the solution seems to be insensitive to data disturbance, which is very important if we solve problems based on measurements. In the first examples the results for disturbed data is of course worse than in the case of exact date. However the error stays at a level similar to a given disturbance. This method is also very effective even in the case of incomplete input data. In the presented examples the temperature measurements are given only for a certain period of time from 6 to 16 seconds. Using the described method, the error of approximation of the Biot number stays at a low level. For the example presented in the paper the approximation of the exact solution was highly satisfactory for a small number of heat polynomials. In the case of exact data, the best results are obtained for 8 Trefftz functions. When we consider noisy data the best results we get using 5 and 7 functions. The problem described in section 4 shows that the Trefftz method gives similar results to other methods presented in literature. It is very important that approximation obtained 
using Trefftz method strictly satisfies the governing equation. Therefore, the results seem to be more correct in physical sense.

\section{References}

[1] Trefftz E., Ein Gegenstueuk zum Ritz'schen Verfahren, Proceedings 2nd International Congres of Applied Mechanics, Zurich 1926, 131-137.

[2] Rosenbloom P.C., Widder D.V., Expansion in terms of heat polynomials and associated functions, Transactions of the American Mathematical Society 1956, 92, 220-266.

[3] Ciałkowski M.J., Frąckowiak A., Heat Functions and Their Application for Solving Heat Transfer and Mechanical Problems, Poznań University of Technology Publishers, Poznań 2000.

[4] Hożejowska S., Kaniowski R., Poniewski M.E., Application of adjustment calculus to the Trefftz method for calculating temperature field of the boiling liquid flowing in a minichannel, International Journal of Numerical Methods for Heat and Fluid Flow 2014, 24, 4, 811-824.

[5] Grysa K., Metody określania liczby Biota i współczynnika przejmowania ciepła, Journal of Theoretical and Applied Mechanics 1982, 20, 71-86.

[6] Bartz D.R., A simple equation for rapid estimation of rocket nozzle convective heat transfer coefficients, Jet Propulsion 1957, 49-51.

[7] Mehta R.C., Solution of the inverse conduction problem, AIAA Journal 1977, 1355-1356.

[8] Mehta R.C., Extention of the solution of inverse conduction problem, International Journal of Heat and Mass Transfer 1979, 1149-1150. 